\title{
Intravenous Thrombolysis for Acute Mild Ischemic Stroke Patients: Higher ABCD2 Score Associated with Better Outcome
}

\section{Runnan Li}

First Affiliated Hospital of Soochow University https://orcid.org/0000-0002-9833-6105

\section{Chunyan Han}

First Affiliated Hospital of Soochow University

\section{Xiuying Cai}

First Affiliated Hospital of Soochow University

\section{Yan Kong}

First Affiliated Hospital of Soochow University

\section{Lulu Zhang}

First Affiliated Hospital of Soochow University

\section{Zhengyang Lu}

First Affiliated Hospital of Soochow University

\section{Xueyun Liu}

First Affiliated Hospital of Soochow University

Qi Fang ( $\square$ fangqi_008@126.com )

First Affiliated Hospital of Soochow University Juehua Zhu

First Affiliated Hospital of Soochow University

\section{Research Article}

Keywords: Prognosis, risk scores, stroke, intravenous thrombolysis

Posted Date: February 5th, 2019

DOI: https://doi.org/10.21203/rs.2.298/v1

License: (c) (i) This work is licensed under a Creative Commons Attribution 4.0 International License. Read Full License 


\section{Abstract}

Background: Up to $30 \%$ of patients with mild ischemic stroke suffer neurologic deterioration. However, optimal medical approaches of such patients remain controversial given the efficacy and safety of intravenous thrombolysis (IVT). The purpose of this study was to evaluate whether patients with acute mild stroke stratified with ABCD2 score (the risk of stroke on basis of age, blood pressure, clinical features, duration of symptoms, and presence of diabetes mellitus) could benefit from IVT.

Methods: Among 3321 patients with a final diagnosis of acute ischemic stroke or transient ischemic attack, we retrospectively included 227 patients identified with acute mild neurologic deficits (National Institution of Health Stroke Scale, NIHSS $\leq 5)$ treated with or without IVT. Odds ratios (OR) with their confidence intervals $(\mathrm{Cl})$ for outcomes between groups were assessed by using multivariable binary logistic regression analyses. And the heterogeneity of treatment effect magnitude for excellent outcome at 90 days (modified Rankin Scale [mRS] 0-1) was estimated in different subgroups.

Results: A total of 227 cases were enrolled, 108 receiving IVT and 119 treated with secondary stroke prevention strategies alone. Patients receiving IVT had more history of atrial fibrillation. At 7 days, 32 (29.6\%) patients with IVT treatment versus 17 (14.3\%) patients not receiving IVT achieved significant improvement ( $\geq 4$-point NIHSS score decrease; OR, 2.57; 95\%Cl, 1.29-5.12; $P=0.007$ ). At 90 days, excellent outcome was achieved in $85(78.7 \%)$ patients treated with IVT versus $78(65.5 \%)$ patients without IVT treatment (OR: $2.95 ; 95 \% \mathrm{Cl}, 1.44-6.03 ; P=0.003)$, especially in those with $\mathrm{ABCD} 2$ score $\geq 4(78.4 \%$ versus 64.6\%; OR, 2.81; 95\% Cl, 1.31-6.03; $P=0.008)$. Besides, 7(6.5\%) IVT-treated patients versus 2 (1.7\%) non-IVTtreated patients developed intracranial hemorrhage $(\mathrm{ICH} ; \mathrm{OR}, 4.05 ; 95 \% \mathrm{Cl}, 0.82-19.96 ; \mathrm{P}=0.085)$, among these only $1(0.9 \%)$ was symptomatic ICH with IVT treatment.

Conclusions: For acute mild ischemic stroke patients, we reassured the safety and especially the efficacy of IVT at 7- and 90-days. Patients with 4 or more of ABCD2 score might benefit more from IVT.

Keywords: Prognosis, risk scores, stroke, intravenous thrombolysis.

\section{Background}

Mild stroke accounts for approximately two thirds of acute ischemic stroke patients in population-based studies[1, 2]. Nevertheless, minor neurological deficiency or rapidly improving symptoms are the most common reasons for withdraw from IVT in otherwise guideline-based eligible patients[3]. Previous data indicated that $30 \%$ of such patients had persistent disability at 90 days[4], and the risk of recurrent stroke at 90 days, 1 year and 5 years were $3.7 \%, 5.1 \%$ and $9.5 \%$, respectively $[5,6]$.

Although the proportion of thrombolytic therapy for mild stroke patients has increased over the past decade, the efficacy and safety of recombinant tissue plasminogen activator (rt-PA) remains controversial. A series of studies suggested patients with mild deficiency could benefit from IVT[7-11]. Currently, the American Stroke Association gives a level I (strong) and Ilb (weak) evidence-based score 
respectively for IVT in patients with mild disabling and nondisabling ischemic stroke symptoms within $3 \mathrm{~h}[12]$. But there were also studies indicating no significance in the efficacy between IVT and antiplatelet therapy in patients with mild stroke[13,14], especially in those with non-disabling deficiency[14]. And the patient treated with IVT was not recommended given antithrombotic therapy within 24 hours, even though an aggravation of the condition. In consideration of the controversial therapeutic decisionmaking, it is necessary to screen high-risk mild stroke patients for IVT.

The purpose of this observational study was to investigate whether patients with acute mild stroke symptoms (NIHSS score $\leq 5$ ) could achieve 7- and 90-day good outcome from intravenous rt-PA therapy by comparing with patients treated with timely secondary stroke prevention strategies not receiving rt-PA. We also hypothesized that ABCD2 score might be related to the prognosis of IVT patients.

\section{Methods}

\section{Study Design}

This study was designed as a retrospective study evaluating the efficacy and safety of IVT administered within 4.5 hours of symptom onset.

This study was approved by the First Affiliated Hospital of Soochow University Institutional Review Board. And the need for patient informed consent was waived by the same ethics committee. Consecutive acute ischemic stroke (AIS) patients with minor-to-mild stroke symptoms (NIHSS score $\leq 5$ ) were enrolled from our hospital between August 2016 and May 2018.

\section{Patients Selection}

Eligible cases were collected using the following inclusion criteria: (1) Clinical diagnosis of AIS or transient ischemic stroke (TIA) with NIHSS score 0 to 5; (2) Age 18 years or older; (3) Time from symptom onset within $4.5 \mathrm{~h}$ for rt-PA group, however, it could be administered up to 24 hours after symptom onset in non-rt-PA group; (4) Available for a telephone interview at 90 days. The exclusion criteria were as follows: (1) Pre-stroke mRS score of $\geq 2$; (2) ICH on baseline computed tomography, and other contraindications to IVT and antithrombotic therapy.

\section{Patient Management}

Participants were divided into two groups according to different approaches about the management of mild stroke patients: (1) rt-PA group: Intravenous rt-PA within 4.5 hours of AIS onset or last known well time $(0.9 \mathrm{mg} / \mathrm{kg}$, maximum dose $90 \mathrm{mg}$ with initial $10 \%$ of total dose given as bolus during 1 minute), followed by appropriate secondary stroke prevention strategies; (2) Non-rt-PA group: Optimal secondary stroke prevention strategies within $24 \mathrm{~h}$ from AIS onset: including initiation of dual antiplatelets among patients with NIHSS $\leq 3$ (clopidogrel $75 \mathrm{mg}$ per day for 90 days + aspirin $100 \mathrm{mg}$ per day for the first 3 weeks); clopidogrel $75 \mathrm{mg}$ / aspirin 100mg per day; or anticoagulation agents in the event of cardioembolism, and other measures such as statins and antihypertensive medication, etc. 


\section{Patient Data Collection}

Baseline demographic and clinical information were collected by experienced stroke neurologists: including age, gender, previous history (hypertension, diabetes mellitus, atrial fibrillation, coronary heart disease, previous stroke or TIA and current smoking), medication history, stroke severity (measured by NIHSS score at admission and 7d), time from symptom onset, blood pressure at baseline, $A B C D_{2}$ score, stroke subtype (assessed using TOAST classification [Trial of Org 10172 in Acute Stroke Treatment])[15], and neurological imaging (collected at baseline, $24-36 \mathrm{~h}, 7 \mathrm{~d}$ or discharge if sooner in stroke unit). The $90 \mathrm{~d}-$ mRS was assessed by telephone follow-up.

\section{Definition of Clinical Information}

All participants were hospitalized in the stroke unit assessed by neurologic specialists. (1) Efficacy outcomes: The achievement of an mRS of 0 to 1 at 90 days was defined as excellent outcome, and an $\mathrm{mRS}$ of 0 as perfect outcome. Significant improvement was referred as complete resolution of the neurologic deficit or an improvement of at least 4 points over baseline NIHSS score[16]. (2) Adverse outcomes: Early neurological deterioration (END) was defied as a NIHSS score increase of 2 or more within 7d after symptom onset excluding any CT- or MRI-documented ICH[17]. sICH was defined as CTbased ICH within 7 days with a NIHSS score increase of at least 4 or death[18]. The recurrent ischemic stroke within 3 months was assessed by stroke specialists via face-to-face interviews or telephone followup.

\section{Statistical Analysis}

Categorical variables are expressed as numbers (\%). Continuous variables are expressed as mean (standard deviation [SD]) in the case of normal distribution, otherwise as median (interquartile range [IQR]), and the normality of distributions was evaluated by histograms and the Shapiro-Wilk test. The between-group differences in baseline characteristics were assessed as follows: continuous variables were compared with the Student $t$ test or Mann-Whitney test as appropriate, while categorical variables were compared with chi-square test (Pearson's $\chi 2$ or continuity correction). ORs with their Cls for efficacy and adverse outcomes were estimated respectively using multivariable binary logistic regression analyses in the whole study group. Moreover, we intended to estimate the heterogeneity of therapeutic effect magnitude for excellent outcome stratified by baseline NIHSS score ( $₫ 3$ versus $\geq 3$ ), stroke subtype (large artery atherosclerosis, cardioembolism, small vessel occlusion and other/undetermined etiology), disabling neurologic deficits (yes versus no), and baseline $A B C D_{2}$ score ( $₫ 4$ versus $\geq 4$ ).

The a-level of significance was $P<0.05$ two-tailed. All analyses were performed using the SPSS software version 25.0 .

\section{Results}

\section{Population and Baseline Characteristics}


Between August 2016 and May 2018, a total of 327 patients with AIS were treated with intravenous rt-PA in stroke unit within 4.5h, among which 111 (33.9\%) with mild neurologic deficit (baseline NIHSS score $\leq 5)$. And 3 were excluded for lost during follow-up, resulting in 108 patients in the rt-PA group.

Meanwhile, 2994 patients were admitted to the unit with AIS not receiving intravenous rt-PA. Among these 119 participants responding to inclusion criteria were enrolled in the non-rt-PA group (Figure 1).

The median age of rt-PA treated patients was 65.5 (IQR, 59-74), and 66 (IQR, 59-74) in rt-PA untreated patients. There were 30 patients $(27.8 \%)$ in rt-PA group and 45 patients $(37.8 \%)$ in non-rt-PA group were female. The median baseline NIHSS score in different medical approaches was both $3($ IQR, 2-4; $P=0.09)$. And the median baseline $A B C D_{2}$ score among two groups was also matched ( 5 versus 5 ; IQR, 4-6 versus $5-6 ; P=0.58)$. Study groups were generally balanced as shown in Table 1 , with the most common medical risk factors being hypertension (71.8\%) and current smoking (34.8\%). However, patients in rt-PA group had a significantly higher proportion of history of atrial fibrillation ( $18.5 \%$ versus. $7.6 \%, P=0.01)$. Among patients with mild stroke, the most common stroke subtype was small vessel occlusion (37.9\%) followed by large artery atherosclerosis (32.6\%), with other/undetermined etiology (15.4\%) and cardioembolism (14.1\%) less frequent.

\section{Efficacy outcomes}

Figure 2 showed the comparisons in efficacy and adverse outcomes between different medical approaches. And multivariable-adjusted associations between covariates and efficacy/adverse outcomes were showed in Table 2. Excellent outcome at 90 days was achieved in $85(78.7 \%)$ patients treated with rt-PA compared with 78 (65.5\%) patients without rt-PA treatment (Figure 2), with an adjusted OR of 2.95 (95\% Cl, 1.44-6.03; $P=0.003$ ) after controlling for the effects of male (OR, 3.2; $95 \% \mathrm{Cl}, 1.60-6.28 ; P=0.001)$, history of diabetes mellitus (OR, $0.34 ; 95 \% \mathrm{Cl}, 0.16-0.70 ; P=0.004)$ and worse baseline NIHSS score (OR, $0.51 ; 95 \% \mathrm{Cl}, 0.38-0.69 ; \mathrm{P}<0.001$ ) (Table 2). Meanwhile, excellent outcome at 90 days achieved by intravenous rt-PA therapy was both observed in different time window from symptom onset ( 0 -3h versus $3-4.5 \mathrm{~h}, 83.9 \%$ versus $73.1 \%, \mathrm{P}=0.17$ ) (data not shown). Similar result was found considering perfect outcome (OR, 2.08; 95\% Cl, 1.17-3.70; $P=0.013)$. Besides, worse stroke severity (OR, $0.66 ; 95 \% \mathrm{Cl}, 0.52-0.83$; $P \otimes 0.001)$ and history of diabetes mellitus $(\mathrm{OR}, 0.48 ; 95 \% \mathrm{Cl}, 0.24-0.97 ; P=0.039)$ were associated with lower rate of perfect outcome. Moreover, we detected significant improvement in $32(29.6 \%)$ patients receiving rt-PA treatment $(\mathrm{OR}, 2.57 ; 95 \% \mathrm{Cl}, 1.29-5.12 ; P=0.007)$, with control for the effects of male (OR, $4.52 ; 95 \% \mathrm{Cl}, 1.78-11.49 ; P=0.002)$ and wore baseline NIHSS score (OR, 0.69; $95 \% \mathrm{Cl}, 0.53-0.91 ; P=0.009)$.

\section{Adverse outcomes}

The rate of END within 7 days was $13.0 \%$ in patients treated with rt-PA, whereas $10.1 \%$ in patients without rt-PA treatment, with no statistically significant difference between two groups $(\mathrm{OR}, 1.33 ; 95 \% \mathrm{Cl}, 0.59$ $3.01 ; P=0.497)$. History of diabetes mellitus was strongly associated with $\mathrm{END}$ at 7 days $(\mathrm{OR}, 3.37 ; 95 \% \mathrm{Cl}$, 1.46-7.78; $P=0.004)$. Any ICH within 7 days, occurred more frequently in patients treated with rt-PA compared with patients without rt-PA treatment $(6.5 \%$ versus $1.7 \%$; OR, $4.05 ; 95 \% \mathrm{Cl}, 0.82-19.96 ; P=0.085)$. 
However, only 1 (0.9\%) was sICH in rt-PA group. Older age, high level of systolic blood pressure at admission and worse stroke severity were associated with any $\mathrm{ICH}$. While the recurrent ischemic stroke in 90 days was less frequent in patients treated with rt-PA compared with the other group $(2.8 \%$ versus $4.2 \%$; $\mathrm{OR}, 0.65 ; 95 \% \mathrm{Cl}, 0.15-2.79 ; P=0.564)$. Only male was strongly associated with lower incidence of recurrent ischemic stroke (OR, $0.064 ; 95 \% \mathrm{Cl}, 0.01-0.53 ; P=0.011)$.

\section{Association Between Subgroups and Excellent Outcome}

In the subgroup analysis stratified by $\mathrm{ABCD}_{2}$ score (Figure 3), excellent outcome was achieved in 80 patients $(78.4 \%)$ receiving intravenous it-PA with $A B C D_{2}$ score $\geq 4$, compared with $73(64.6 \%)$ patients not receiving intravenous rt-PA with $A B C D_{2}$ score $\geq 4(O R, 2.81 ; 95 \% \mathrm{Cl}, 1.31-6.03 ; P=0.008)$. For patients with $A B C D_{2}$ score $\mathbb{4}$, no significant of treatment effect was observed between two groups. Excellent outcome was achieved in both subgroups of baseline NIHSS score with significant difference. However, we detected no significant heterogeneity of therapeutic effect magnitude for excellent outcome stratified by stroke subtype and disabling neurologic deficits.

\section{Results}

\section{Population and Baseline Characteristics}

Between August 2016 and May 2018, a total of 327 patients with AIS were treated with intravenous rt-PA in stroke unit within 4.5h, among which 111 (33.9\%) with mild neurologic deficit (baseline NIHSS score $\leq 5)$. And 3 were excluded for lost during follow-up, resulting in 108 patients in the rt-PA group.

Meanwhile, 2994 patients were admitted to the unit with AIS not receiving intravenous rt-PA. Among these 119 participants responding to inclusion criteria were enrolled in the non-rt-PA group (Figure 1).

The median age of rt-PA treated patients was 65.5 (IQR, 59-74), and 66 (IQR, 59-74) in rt-PA untreated patients. There were 30 patients $(27.8 \%)$ in rt-PA group and 45 patients $(37.8 \%)$ in non-rt-PA group were female. The median baseline NIHSS score in different medical approaches was both 3 (IQR, 2-4; $P=0.09)$. And the median baseline $A B C D_{2}$ score among two groups was also matched ( 5 versus 5 ; IQR, 4-6 versus $5-6 ; P=0.58)$. Study groups were generally balanced as shown in Table 1 , with the most common medical risk factors being hypertension $(71.8 \%)$ and current smoking (34.8\%). However, patients in rt-PA group had a significantly higher proportion of history of atrial fibrillation ( $18.5 \%$ versus. $7.6 \%, P=0.01)$. Among patients with mild stroke, the most common stroke subtype was small vessel occlusion (37.9\%) followed by large artery atherosclerosis (32.6\%), with other/undetermined etiology (15.4\%) and cardioembolism (14.1\%) less frequent.

\section{Efficacy outcomes}

Figure 2 showed the comparisons in efficacy and adverse outcomes between different medical approaches. And multivariable-adjusted associations between covariates and efficacy/adverse outcomes were showed in Table 2. Excellent outcome at 90 days was achieved in 85 (78.7\%) patients treated with 
rt-PA compared with 78 (65.5\%) patients without rt-PA treatment (Figure 2), with an adjusted OR of 2.95 $(95 \% \mathrm{Cl}, 1.44-6.03 ; P=0.003)$ after controlling for the effects of male (OR, 3.2; $95 \% \mathrm{Cl}, 1.60-6.28 ; P=0.001)$, history of diabetes mellitus (OR, $0.34 ; 95 \% \mathrm{Cl}, 0.16-0.70 ; P=0.004)$ and worse baseline NIHSS score (OR, $0.51 ; 95 \% \mathrm{Cl}, 0.38-0.69 ; \mathrm{P}<0.001$ ) (Table 2). Meanwhile, excellent outcome at 90 days achieved by intravenous rt-PA therapy was both observed in different time window from symptom onset (0-3h versus $3-4.5 \mathrm{~h}, 83.9 \%$ versus $73.1 \%, \mathrm{P}=0.17$ ) (data not shown). Similar result was found considering perfect outcome (OR, 2.08; 95\% Cl, 1.17-3.70; $P=0.013)$. Besides, worse stroke severity (OR, $0.66 ; 95 \% \mathrm{Cl}, 0.52-0.83$; $P \otimes 0.001)$ and history of diabetes mellitus $(\mathrm{OR}, 0.48 ; 95 \% \mathrm{Cl}, 0.24-0.97 ; P=0.039)$ were associated with lower rate of perfect outcome. Moreover, we detected significant improvement in $32(29.6 \%)$ patients receiving rt-PA treatment $(\mathrm{OR}, 2.57 ; 95 \% \mathrm{Cl}, 1.29-5.12 ; P=0.007)$, with control for the effects of male (OR, $4.52 ; 95 \% \mathrm{Cl}, 1.78-11.49 ; P=0.002)$ and wore baseline NIHSS score (OR, $0.69 ; 95 \% \mathrm{Cl}, 0.53-0.91 ; P=0.009)$.

\section{Adverse outcomes}

The rate of END within 7 days was $13.0 \%$ in patients treated with rt-PA, whereas $10.1 \%$ in patients without rt-PA treatment, with no statistically significant difference between two groups $(\mathrm{OR}, 1.33 ; 95 \% \mathrm{Cl}, 0.59$ $3.01 ; P=0.497)$. History of diabetes mellitus was strongly associated with $\mathrm{END}$ at 7 days $(\mathrm{OR}, 3.37 ; 95 \% \mathrm{Cl}$, 1.46-7.78; $P=0.004$ ). Any ICH within 7 days, occurred more frequently in patients treated with it-PA compared with patients without rt-PA treatment $(6.5 \%$ versus $1.7 \%$; OR, $4.05 ; 95 \% \mathrm{Cl}, 0.82-19.96 ; P=0.085)$. However, only 1 (0.9\%) was sICH in rt-PA group. Older age, high level of systolic blood pressure at admission and worse stroke severity were associated with any $\mathrm{ICH}$. While the recurrent ischemic stroke in 90 days was less frequent in patients treated with rt-PA compared with the other group $(2.8 \%$ versus $4.2 \%$; $\mathrm{OR}, 0.65 ; 95 \% \mathrm{Cl}, 0.15-2.79 ; P=0.564)$. Only male was strongly associated with lower incidence of recurrent ischemic stroke (OR, $0.064 ; 95 \% \mathrm{Cl}, 0.01-0.53 ; P=0.011)$.

\section{Association Between Subgroups and Excellent Outcome}

In the subgroup analysis stratified by $A B C D_{2}$ score (Figure 3), excellent outcome was achieved in 80 patients $(78.4 \%)$ receiving intravenous it-PA with $A B C D_{2}$ score $\geq 4$, compared with $73(64.6 \%)$ patients not receiving intravenous rt-PA with $A B C D_{2}$ score $\geq 4(O R, 2.81 ; 95 \% \mathrm{Cl}, 1.31-6.03 ; P=0.008)$. For patients with $A B C D_{2}$ score $\mathbb{4}$, no significant of treatment effect was observed between two groups. Excellent outcome was achieved in both subgroups of baseline NIHSS score with significant difference. However, we detected no significant heterogeneity of therapeutic effect magnitude for excellent outcome stratified by stroke subtype and disabling neurologic deficits.

\section{Discussion}

Our study implied a high proportion of $78.7 \%$ acute mild ischemic stroke (NIHSS score $\leq 5$ ) patients receiving intravenous rt-PA within 4.5 hours achieved excellent outcome (mRS 0-1) at 90 days, and $29.7 \%$ with significant improvement at 7 days, indicating IVT being effective for patients with mild deficits. And 
the encouraging results were both detected in the $0-3 \mathrm{~h}(83.9 \%)$ and $3-4.5 \mathrm{~h}(73.1 \%)$ time windows. Notably, we found that patients with mild stroke whose $A B C D_{2}$ score $\geq 4$ could benefit more from IVT.

A series of randomized studies $[7,14]$, systematic review[8-10] and observational cohorts $[11,13]$ indicated controversial results regarding the efficacy of IVT among patients with acute mild ischemic stroke. The post hoc analysis in a rigorously selected sample (restricted to 106 participants with a baseline NIHSS score $\leq 5$ within 3 hours from symptom onset) of the third International Stroke Trial (IST-3) suggested encouraging results of intravenous it-PA efficacy in mild ischemic stroke (84\% rt-PA versus $65 \%$ control; adjusted odds ratio, 3.31; $95 \% \mathrm{Cl}, 1.24-8.79 ; P=0.03)$ [7], which is consistent with our study and most post previous data[8-11]. Nevertheless, a large observational cohort from China suggested that intravenous rtPA might potentially benefit patients with NIHSS score $\leq 5$ within 4.5 hours from symptom onset, with no statistical significance (76\% rt-PA versus $69.5 \%$ control; odds ratio, $1.48 ; 95 \% \mathrm{Cl}, 0.91-2.43 ; P=0.12)[13]$. The difference in efficacy of IVT might be associated with the different time window selection, various definition of mild stroke and better secondary stroke prevention measurements. Currently, the preliminary results of Potential of rtPA for Ischemic Strokes with Mild Symptoms (PRISMS)[14], a double-blind, multicenter, randomized controlled trial, revealed that likelihood of excellent outcome at 90 days didn't increase among mild nondisabling AIS patients receiving IVT treatment, yet a higher risk of complication of sICH. However, the early termination with only 313 participants enrolled might preclude the definitive conclusions. Our study didn't detect significant difference for excellent outcome stratified by disabling deficiency in two medical approaches either. The disabling deficit is commonly defined as follows: complete hemianopia (NIHSS-3 $\geq 2$ ), severe aphasia (NIHSS-9 $\geq 2$ ), neglect NIHSS-11 $\geq 1$ ), limb weakness that cannot resist gravity (NIHSS-5/6 $\geq 2$ ), functional impairment with NIHSS $>5$, or any potential disabling deficit judged by experienced physician[14]. Nevertheless, the NIHSS scale may not be adequate to evaluate severity of mild stroke, especially in posterior circulation AIS patients[19], hence considerable patients are regarded as nondisabling deficiency, resulting in unfavorable outcome. So accurate description of disabling neurological deficits is warranted urgently to screen high-risk mild AIS patients.

As for the safety of intravenous rt-PA in mild stroke, we detected 7 (6.5\%) receiving intravenous rt-PA patients occurred ICH within 7 days compared with the lower rate of $1.7 \%$ in non-rt-PA group, yet among these only $1(0.9 \%)$ developed sICH from rt-PA group, lower than the proportion of $1.8 \%-4.1 \%$ previous studies had reported[14, 20,21]. The significant association between post-IVT ICH with older age, worse stroke severity and higher admission glucose level was reported in a systematic review from 55 studies[22], similar with our study data. The incidence of sICH in a large single-center cohort[23] stratified by baseline NIHSS score ( $\leq 6$ versus $₫ 6$ ) was $2.0 \%$ and $8.1 \%(P \otimes 0.001)$, respectively, while the difference was not detected with increasing NIHSS score within the range of 0 to $5(P=0.51)$ [20], indicating that intravenous rt-PA is relatively safe but not risk-free in mild stroke patients.

Our study demonstrated that acute mild stroke with $A B C D_{2}$ score $\geq 4$, a fresh perspective to screen appropriate patients, might potentially benefit more from intravenous it-PA treatment. $A B C D_{2}$ score was intended to aid clinical management and estimate the risk of stroke recurrence in patients with TIA and minor stroke[24]. In addition, $\mathrm{ABCD}_{3}$-I score (range $0-13$, addition of dual TIA within 7 days, ipsilateral 
carotid artery stenosis $\geq 50 \%$ and positive brain imaging) showed better validation for prediction of early and 90-day stroke recurrent risk, with clinical presentation (C), symptom duration (D) and cerebral/carotid imaging (I) being the most essential components[25-27]. Recent trial revealed the $A B C D_{2}$ score of 4 and more in TIA or minor stroke was significantly associated with longer-term risk of another stroke[6]. Also, these scores were used to select patients for intensive therapy[28, 29]. However, it remains unclear regarding the validation of the risk scores to screen high-risk candidates for intravenous it-PA among mild stroke patients, so that further investigation in randomized trials or larger observational studies is needed. Besides, in clinical practice, physicians can as well refer to the stroke etiology of large artery atherosclerosis[13] and presentation of disability[12] to assist thrombolytic decision-making.

Thus, further investigations are warranted. Mild and Rapidly Improving Stroke Study (MaRISS; observational trail; NCT02072681) and Antiplatelet vs Rt-PA for Acute Mild Ischemic Stroke (ARAMIS; randomized, placebo-controlled trial; NCT03661411) are two ongoing trails investigating the safety and particularly the efficacy of intravenous rt-PA in patients with acute mild stroke.

The non-randomized design due to retrospective study is the most notable limitation of our research. Although, the baseline characteristics between two groups were matched generally, with significant difference only in history of atrial fibrillation, the well-known limitations of observational study still not be avoided. And the small sample size of 227 enrolled subjects in this study limited the power to assess the efficacy and safety of IVT among mild stroke patients. In addition, majorities of our enrolled participants had 4 points or more on baseline $A B C D_{2}$ score, which might cause bias and undermine the results. Considering the inadequacy to generalize the results of our research to nation-wide population with mild stroke, well-designed multicenter randomized clinical trials are needed.

\section{Conclusions}

A substantial proportion of patients deemed mild stroke (NIHSS $\leq 5)$ with intravenous rt-PA therapy has excellent outcome both at 7- and 90-days, especially among patients with 4 or more of baseline $A B C D_{2}$ score. While, accurate identification of high-risk stroke is so essential to screen patients with mild deficiency for IVT, that further researches are warranted. In conclusion, given the significant percentage of excellent outcome and low risk of $\mathrm{sICH}$, our results reassure the efficacy and safety of IVT therapy for acute mild ischemic stroke patients.

\section{Abbreviations}

$A B C D_{2}$ score: The risk of stroke on basis of age, blood pressure, clinical features, duration of symptoms, and presence of diabetes mellitus; $\mathrm{ABCD}_{3}$-I score: Addition of dual symptom onsets within 7 days, ipsilateral carotid artery stenosis $\geq 50 \%$ and positive brain imaging on the basis of $A B C D_{2}$ score; AIS: Acute ischemic stroke; ARAMIS: Antiplatelet vs rt-PA for acute mild ischemic stroke; Cl: Confidence interval; END: Early neurological deterioration ICH: Intracranial hemorrhage; IQR: Interquartile range; IVT: Intravenous thrombolysis; MaRISS: Mild and rapidly improving stroke study; mRS: Modified Rankin scale; 
NIHSS: National Institutes of Health Stroke Scale; OR: Odds ratio; PRISMS: Potential of rtPA for ischemic strokes with mild symptoms; rt-PA: Recombinant tissue plasminogen activator; SD: Standard deviation; sICH: Symptomatic intracranial hemorrhage; TIA: Transient ischemic stroke; TOAST: Trial of org 10172 in acute stroke treatment.

\section{Declarations}

\section{Ethics approval and consent to participate}

The use of data analysis was legally approved by the at the First Affiliated Hospital of Soochow University Institutional Review Board. This research was performed in accordance with the tenets of the Declaration of Helsinki as amended in 2008. The need for patient informed consent was waived by the same ethics committee.

\section{Consent for publication}

Not applicable.

\section{Availability of data and material}

The datasets used and analyzed during the current study are available from the corresponding author on reasonable request.

\section{Competing interests}

The authors declare that they have no competing interests.

\section{Funding}

This work was supported by grant from the National key R\&D Program of China (No.2017YFC0114300), the National Natural Science Foundation of China (No. 81601011), the Natural Science Foundation of Jiangsu Province (No. BK20160345) and Suzhou Government (No. KJXW2016002). The role of the funding body was limited to finance the first author for collection, analysis and interpretation of data and revision of the article during her Masters.

\section{Authors' contributions}

QF, XC and YK designed this study. RL and $\mathrm{CH}$ collected and analyzed the patient data, and were the major contributor in writing the manuscript. JZ, LZ, RL, CH, ZL and XL conducted the clinical assessments and follow-up of participants. All authors contributed to creating this manuscript and improved the final version.

\section{Acknowledgements}

We thank Mingzhi Zhang for the help of statistical analysis. 


\section{References}

1. Reeves M, Khoury J, Alwell K, Moomaw C, Flaherty M, Woo D, et al. Distribution of National Institutes of Health stroke scale in the Cincinnati/Northern Kentucky Stroke Study. Stroke. 2013;44(11):3211-3.

2. Kim BJ, Park JM, Kang K, Lee SJ, Ko Y, Kim JG, et al. Case characteristics, hyperacute treatment, and outcome information from the clinical research center for stroke-fifth division registry in South Korea. J Stroke. 2015;17(1):38-53.

3. Messe SR, Khatri P, Reeves MJ, Smith EE, Saver JL, Bhatt DL, et al. Why are acute ischemic stroke patients not receiving IV tPA? Results from a national registry. Neurology. 2016;87(15):1565-74.

4. Khatri P, Conaway MR, Johnston KC. Ninety-day outcome rates of a prospective cohort of consecutive patients with mild ischemic stroke. Stroke. 2012;43(2):560-2.

5. Amarenco P, Lavallee PC, Labreuche J, Albers GW, Bornstein NM, Canhao P, et al. One-Year Risk of Stroke after Transient Ischemic Attack or Minor Stroke. N Engl J Med. 2016;374(16):1533-42.

6. Amarenco P, Lavallee PC, Monteiro Tavares L, Labreuche J, Albers GW, Abboud H, et al. Five-Year Risk of Stroke after TIA or Minor Ischemic Stroke. N Engl J Med. 2018;378(23):2182-90.

7. Khatri P, Tayama D, Cohen G, Lindley RI, Wardlaw JM, Yeatts SD, et al. Effect of Intravenous Recombinant Tissue-Type Plasminogen Activator in Patients With Mild Stroke in the Third International Stroke Trial-3: Post Hoc Analysis. Stroke. 2015;46(8):2325-7.

8. You S, Saxena A, Wang X, Tan W, Han Q, Cao Y, et al. Efficacy and safety of intravenous recombinant tissue plasminogen activator in mild ischaemic stroke: a meta-analysis. Stroke Vasc Neurol. 2018;3(1):22-7.

9. Lees KR, Emberson J, Blackwell L, Bluhmki E, Davis SM, Donnan GA, et al. Effects of Alteplase for Acute Stroke on the Distribution of Functional Outcomes: A Pooled Analysis of 9 Trials. Stroke. 2016;47(9):2373-9.

10. Emberson J, Lees KR, Lyden P, Blackwell L, Albers G, Bluhmki E, et al. Effect of treatment delay, age, and stroke severity on the effects of intravenous thrombolysis with alteplase for acute ischaemic stroke: a meta-analysis of individual patient data from randomised trials. The Lancet. 2014;384(9958):1929-35.

11. Greisenegger S, Seyfang L, Kiechl S, Lang W, Ferrari J, Austrian Stroke Unit Registry C. Thrombolysis in patients with mild stroke: results from the Austrian Stroke Unit Registry. Stroke. 2014;45(3):765-9.

12. Powers WJ, Rabinstein AA, Ackerson T, Adeoye OM, Bambakidis NC, Becker K, et al. 2018 Guidelines for the Early Management of Patients With Acute Ischemic Stroke: A Guideline for Healthcare Professionals From the American Heart Association/American Stroke Association. Stroke. 2018;49(3):e46-e110.

13. Chen W, Pan Y, Zhao X, Liu L, Li H, Liao X, et al. Intravenous Thrombolysis in Chinese Patients with Different Subtype of Mild Stroke: Thrombolysis in Patients with Mild Stroke. Sci Rep. 2017;7(1):2299.

14. Khatri P, Kleindorfer DO, Devlin T, Sawyer RN, Jr., Starr M, Mejilla J, et al. Effect of Alteplase vs Aspirin on Functional Outcome for Patients With Acute Ischemic Stroke and Minor Nondisabling Neurologic 
Deficits: The PRISMS Randomized Clinical Trial. Jama. 2018;320(2):156-66.

15. Adams HP, Jr., Bendixen BH, Kappelle LJ, Biller J, Love BB, Gordon DL, et al. Classification of subtype of acute ischemic stroke. Definitions for use in a multicenter clinical trial. TOAST. Trial of Org 10172 in Acute Stroke Treatment. Stroke. 1993;24(1):35-41.

16. Tissue plasminogen activator for acute ischemic stroke. N Engl J Med. 1995;333(24):1581-7.

17. Lee S-J, Hong JM, Lee SE, Kang DR, Ovbiagele B, Demchuk AM, et al. Association of fibrinogen level with early neurological deterioration among acute ischemic stroke patients with diabetes. BMC Neurol. 2017;17(1).

18. de Los Rios la Rosa F, Khoury J, Kissela BM, Flaherty ML, Alwell K, Moomaw CJ, et al. Eligibility for Intravenous Recombinant Tissue-Type Plasminogen Activator Within a Population: The Effect of the European Cooperative Acute Stroke Study (ECASS) III Trial. Stroke. 2012;43(6):1591-5.

19. Yoshimura S, Lindley RI, Carcel C, Sato S, Delcourt C, Wang X, et al. NIHSS cut point for predicting outcome in supra- vs infratentorial acute ischemic stroke. Neurology. 2018;91(18):e1695-e701.

20. Romano JG, Smith EE, Liang L, Gardener H, Camp S, Shuey L, et al. Outcomes in mild acute ischemic stroke treated with intravenous thrombolysis: a retrospective analysis of the Get With the GuidelinesStroke registry. JAMA Neurol. 2015;72(4):423-31.

21. Choi JC, Jang MU, Kang K, Park JM, Ko Y, Lee SJ, et al. Comparative Effectiveness of Standard Care With IV Thrombolysis Versus Without IV Thrombolysis for Mild Ischemic Stroke. Journal of the American Heart Association. 2015;4(1).

22. Whiteley WN, Slot KB, Fernandes P, Sandercock P, Wardlaw J. Risk factors for intracranial hemorrhage in acute ischemic stroke patients treated with recombinant tissue plasminogen activator: a systematic review and meta-analysis of 55 studies. Stroke. 2012;43(11):2904-9.

23. Strbian D, Piironen K, Meretoja A, Sairanen T, Putaala J, Tiainen M, et al. Intravenous thrombolysis for acute ischemic stroke patients presenting with mild symptoms. Int J Stroke. 2013;8(5):293-9.

24. Rothwell PM, Giles MF, Flossmann E, Lovelock CE, Redgrave JNE, Warlow CP, et al. A simple score $(A B C D)$ to identify individuals at high early risk of stroke after transient ischaemic attack. The Lancet. 2005;366(9479):29-36.

25. Merwick Á, Albers GW, Amarenco P, Arsava EM, Ay H, Calvet D, et al. Addition of brain and carotid imaging to the ABCD2 score to identify patients at early risk of stroke after transient ischaemic attack: a multicentre observational study. The Lancet Neurology. 2010;9(11):1060-9.

26. Song B, Fang H, Zhao L, Gao Y, Tan S, Lu J, et al. Validation of the ABCD3-I score to predict stroke risk after transient ischemic attack. Stroke. 2013;44(5):1244-8.

27. Knoflach M, Lang W, Seyfang L, Fertl E, Oberndorfer S, Daniel G, et al. Predictive value of ABCD2 and ABCD3-I scores in TIA and minor stroke in the stroke unit setting. Neurology. 2016;87(9):861-9.

28. Wang Y, Wang Y, Zhao X, Liu L, Wang D, Wang C, et al. Clopidogrel with aspirin in acute minor stroke or transient ischemic attack. N Engl J Med. 2013;369(1):11-9. 
29. Johnston SC, Easton JD, Farrant M, Barsan W, Battenhouse H, Conwit R, et al. Platelet-oriented inhibition in new TIA and minor ischemic stroke (POINT) trial: rationale and design. Int J Stroke. 2013;8(6):479-83.

\section{Tables}

Table 1 Baseline Characteristics of Patients with Mild Stroke According to therapeutic approach.

\begin{tabular}{|c|c|c|c|}
\hline Characteristics & $\begin{array}{l}\text { rt-PA } \\
(\mathrm{n}=108)\end{array}$ & $\begin{array}{l}\text { Non-rt-PA } \\
(\mathrm{n}=119) \\
\end{array}$ & $P$ value \\
\hline Age, y, median (IQR) & $65.5(59-74)$ & $66(59-74)$ & 0.83 \\
\hline Female, n (\%) & $30(27.8)$ & $45(37.8)$ & 0.11 \\
\hline \multicolumn{4}{|l|}{ Medical history, n (\%) } \\
\hline Hypertension & $78(72.2)$ & $85(71.4)$ & 0.89 \\
\hline Diabetes mellitus & $31(28.7)$ & $28(23.5)$ & 0.38 \\
\hline Atrial Fibrillation & $20(18.5)$ & $9(7.6)$ & 0.01 \\
\hline Coronary heart disease & $8(7.4)$ & $10(8.4)$ & 0.78 \\
\hline Previous stroke/TIA & $16(14.8)$ & $17(14.3)$ & 0.91 \\
\hline Current smoking & $42(38.9)$ & $37(31.1)$ & 0.22 \\
\hline \multicolumn{4}{|l|}{ Medications prior to onset, $\mathrm{n}(\%)$} \\
\hline Antihypertension agents & $62(57.4)$ & $69(58.0)$ & 0.93 \\
\hline Antidiabetic agents & $24(22.2)$ & $21(17.6)$ & 0.39 \\
\hline Antiplatelet agents & 15(13.9) & $12(10.1)$ & 0.38 \\
\hline Anticoagulant agents & $3(2.8)$ & $2(1.7)$ & 0.67 \\
\hline Baseline NIHSS score, median (IQR) & $3(2-4)$ & $3(2-4)$ & 0.09 \\
\hline Baseline ABCD2 score, median (IQR) & $5(4-6)$ & $5(5-6)$ & 0.58 \\
\hline Stroke subtype, n (\%) & & & 0.25 \\
\hline Large artery atherosclerosis & $36(33.3)$ & $38(51.4)$ & \\
\hline Cardioembolism & $20(18.5)$ & $12(10.1)$ & \\
\hline Small vessel occlusion & $38(35.2)$ & $48(40.3)$ & \\
\hline Other/Undetermined etiology & $14(13.0)$ & $21(17.6)$ & \\
\hline $\begin{array}{l}\text { Systolic blood pressure, } \mathrm{mmHg} \text {, } \\
(\text { mean } \pm \mathrm{SD})\end{array}$ & $153.8 \pm 21.2$ & $149.3 \pm 18.9$ & 0.09 \\
\hline $\begin{array}{l}\text { Diastolic blood pressure, } \mathrm{mmHg} \text {, } \\
(\text { mean } \pm \mathrm{SD})\end{array}$ & $85.5 \pm 13.3$ & $83.8 \pm 12.1$ & 0.329 \\
\hline
\end{tabular}

Abbreviations: ABCD2 score (age, blood pressure, clinical features, duration of symptoms, and presence of diabetes mellitus; range, 0 to 7, with higher scores indicating a higher risk of stroke); IQR, interquartile range; mRS, modified Rankin Scale; NIHSS, National Institutes of Health Stroke Scale; rt-PA, recombinant tissue plasminogen activator; SD, Standard Deviation; ang TIA, transient ischemic stroke.

Table 2. Multivariable-adjusted Associations Between Covariates and Clinical Outcomes. 


\begin{tabular}{lll}
\hline Outcomes & variables & OR (95\%CI) \\
\hline Excellent outcome & Intravenous rt-PA & $2.95(1.44-6.03)$ \\
& Male & $3.17(1.60-6.28)$ \\
\cline { 2 - 3 } & Diabetes mellitus & $0.34(0.16-0.70)$ \\
\cline { 2 - 3 } Perfect outcome & Baseline NIHSS score, per 1 point & $0.51(0.38-0.69)$ \\
\cline { 2 - 3 } & Intravenous rt-PA & $2.08(1.17-3.70)$ \\
& Baseline NIHSS score, per 1 point & $0.66(0.52-0.83)$ \\
\cline { 2 - 3 } Significant improvement & Diabetes mellitus & $0.48(0.24-0.97)$ \\
\cline { 2 - 3 } & Intravenous rt-PA & $2.57(1.29-5.12)$ \\
& Male & $4.52(1.78-11.49)$ \\
\cline { 2 - 3 } END & Baseline NIHSS score, per 1 point & $0.69(0.53-0.91)$ \\
\cline { 2 - 3 } Any ICH & Diabetes mellitus & $3.37(1.46-7.78)$ \\
\cline { 2 - 3 } & Baseline NIHSS score, per 1 point & $3.81(1.37-10.59)$ \\
& Age, per 1 year & $1.26(1.06-1.49)$ \\
\cline { 2 - 3 } & Systolic blood pressure, per 1 mmHg & $1.10(1.02-1.19)$ \\
\cline { 2 - 3 } Recurrent ischemic stroke & Large artery atherosclerosis & $0.01\left(1.06 \times 10^{-4}-0.27\right)$ \\
\cline { 2 - 3 } & Male & $0.06(0.01-0.53)$ \\
\hline
\end{tabular}

Abbreviations: CI, confidence interval; END, early neurological deterioration; ICH, intracranial hemorrhage; NIHSS, National Institutes of Health Stroke Scale; OR, odds ratio; and rt-PA, recombinant tissue plasminogen activator.

Figures

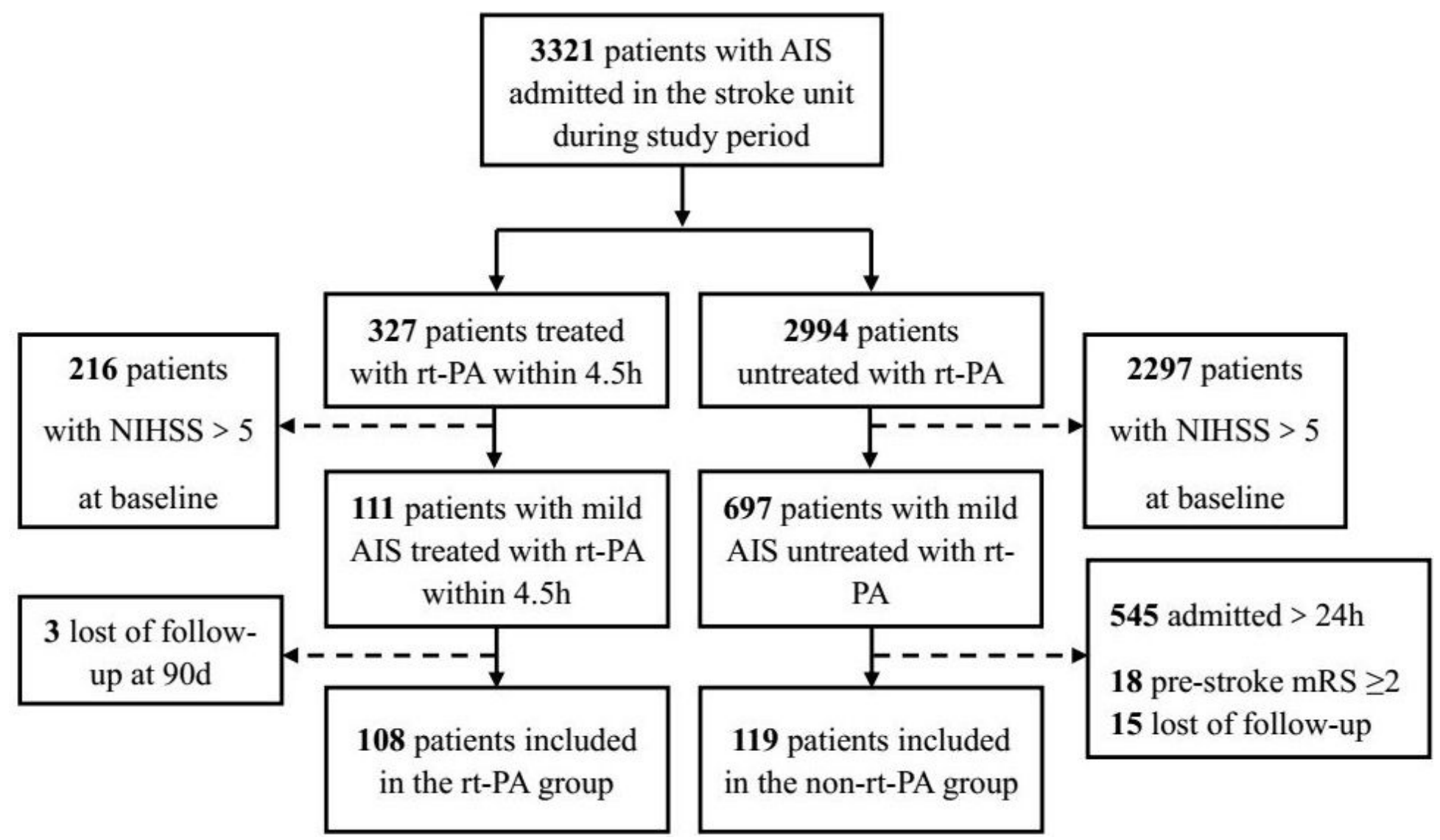


Figure 1

The Flowchart of The Study. Abbreviations: AIS, acute ischemic stroke; mRS, modified Rankin Scale; NIHSS, National Institutes of Health Stroke Scale; rt-PA, recombinant tissue plasminogen activator.

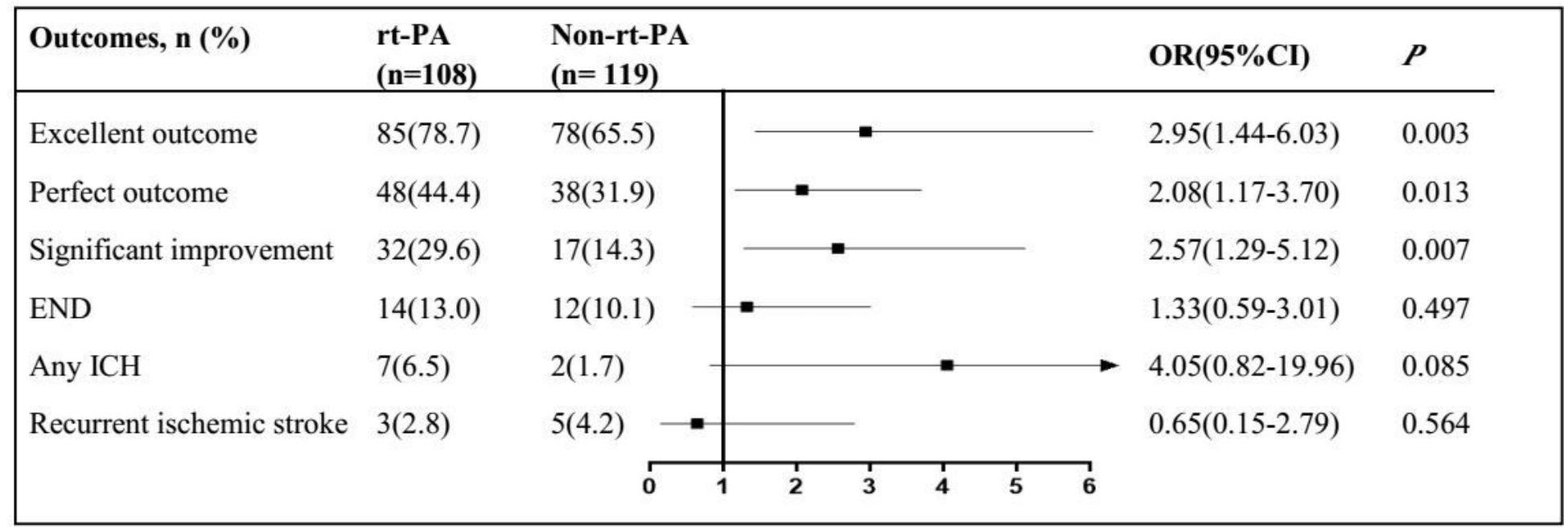

\section{Figure 2}

Comparisons of Clinical Outcomes with Mild Stroke Between Different Medical Approaches.

Abbreviations: $\mathrm{Cl}$, confidence interval; END, early neurological deterioration; ICH, intracranial hemorrhage; and $\mathrm{OR}$, odds ratio.

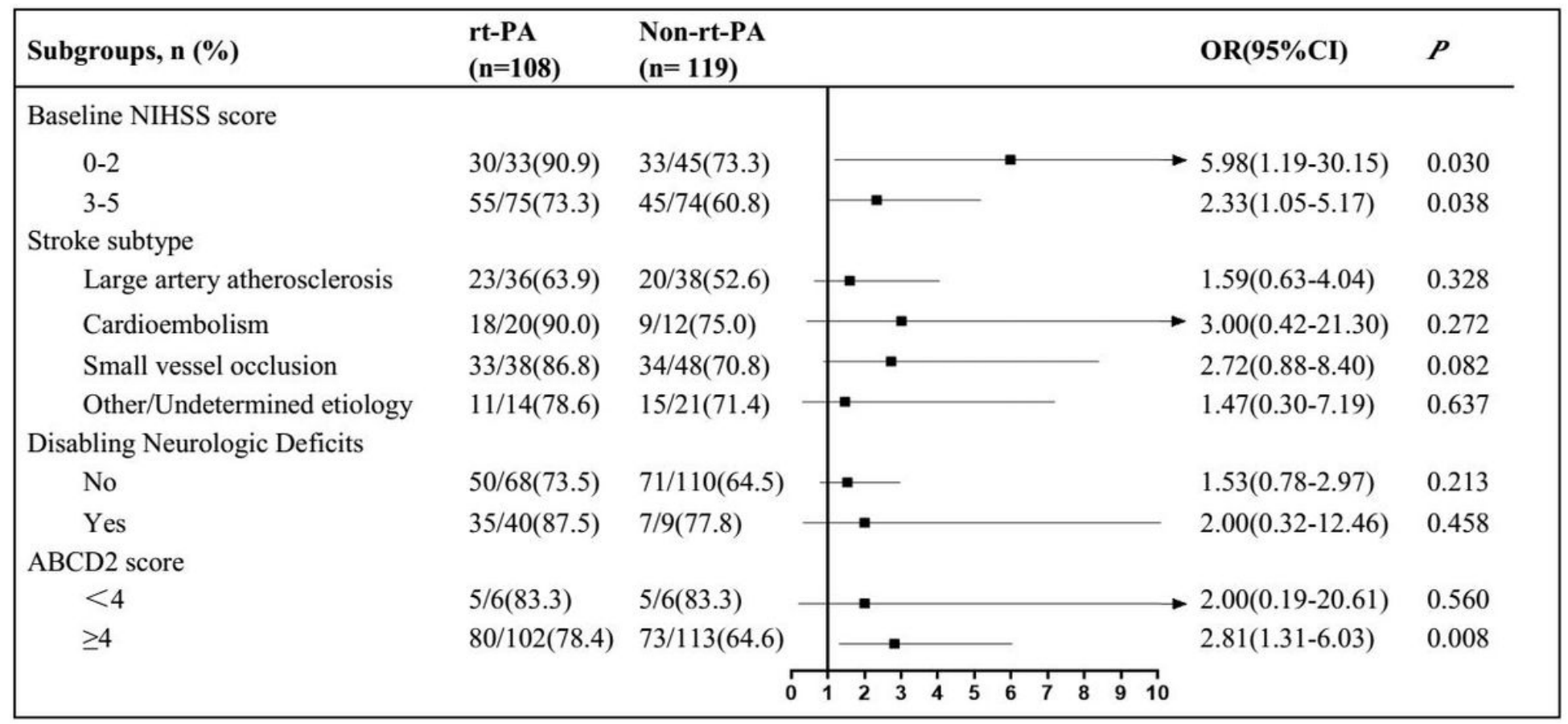

\section{Figure 3}

Subgroup Analysis with Mild Stroke for Excellent Outcome. Abbreviations: ABCD2 score (age, blood pressure, clinical features, duration of symptoms, and presence of diabetes mellitus; range, 0 to 7 , with higher scores indicating a higher risk of stroke); $\mathrm{Cl}$, confidence interval; and $\mathrm{OR}$, odds ratio. 